\title{
LA EXTRACCIÓN DE CAUCHO EN COSTA RICA
}

\author{
Rubber Extraction in Costa Rica
}

\author{
Luis Alberto Sell Biasetti \\ Educador pensionado. Costa Rica \\ sellchaves77@gmail.com
}

Recibido: 09092020

Aprobado: 21092020

El traductor es educador e investigador. Licenciado en Docencia, Licenciado en Administración Educativa. y egresado de la carrera de Doctorado en Ciencias de la Educación de la Universidad Católica de Costa Rica, (tesis pendiente). Trabajó en el Ministerio de Educación Pública, la Universidad de San José y el Banco Central de Costa Rica. Es miembro de la Academia Costarricense de Ciencias Genealógicas y de la Asociación de Genealogía e Historia de Costa Rica (ASOGEHI), de la que fue su presidente.

\section{RESUMEN}

El presente artículo fue publicado en el Wochenschrift des Vereines zur Beförderung des Gartenbaues in den Königlich Preussischen Staaten für Gärtnerei und Pflanzenkunde (Semanario de la Asociación para la Promoción de la Horticultura en los Estados Reales de Prusia para la Jardinería y la Botánica), Berlín, Alemania, el 9 de agosto de 1862, bajo el título "Die Kautschukgewinnung in Costa-Rica". El autor fue el botánico Eduard Sell, alemán radicado entonces en San Ramón de Alajuela, Costa Rica.

Palabras clave: Extracción; caucho; botánica; mercado; negocios; materias primas; alemanes; investigación; Costa Rica.

\section{ABSTRACT}

This article was published in the Weekly of the Association for the Promotion of Horticulture in the Royal States of Prussia for Gardening and Botany (Wochenschrift des Vereines zur Beförderung des Gartenbaues in den Königlich Preussischen Staaten für Gärtnerei und Pflanzenkunde), Berlin, Germany, on August 9, 1862. The author was the botanist Eduard Sell, german based then in San Ramón de Alajuela, Costa Rica.

Keywords: Rubber, extraction; botanics; Market; business; raw materials; germans; research; Costa Rica

\section{Introducción. Relevancia histórica del artículo}

Durante la administración del segundo presidente constitucional de Costa Rica, Juan Rafael Mora Porras (1849-1860), se emitieron varias normativas tendientes a conservar los recursos naturales de Costa Rica. La documentación de la riqueza natural del país por parte de notables científicos extranjeros, tales como Alexander von Humboldt, Julián Carmiol (Carnighol), Karl Hoffman, Anders Oersted, Alexander von Frantzius, Augustus Endrés y otros, así como sus colaboradores, fue posible, entre otros factores, gracias al apoyo que el gobierno de don Juanito dio a la actividad científica. Hay una importante 
investigación al respecto del Dr. don Luko Hilje Quirós, Biólogo, Doctor en Entomología y Profesor Emérito del CATIE ${ }^{1}$.

\section{Sobre el autor}

Eduard Sell nació el 16 de noviembre de 1834 en Ebersdorf, Principado Reuss Línea Menor (Alemania), hijo de Johnn Christieb Heinrich Sell, de Ebersdorf, y Louise Caroline Modes, natural de Gera, Principado Reuss Línea Menor (Alemania).

Fue Inspector en el Real Jardín Zoológico de Bruselas. Este jardín zoológico fue vendido en 1861 al Municipio de la ciudad, a cuyo cargo estuvo hasta cerrar sus puertas al público en 1878, y declarar la bancarrota.

El 19 de octubre de 1861 Eduard viajó a América Central y del Sur con fines botánicos. El anuncio del viaje aparece publicado en 2 periódicos de la época y 2 revistas especializadas, a saber:

1. Illustriert Zeitung (Periódico llustrado), de Liepzing, del sábado 2 de noviembre de 1861. N957. Pg. 319, Sección de historia natural y viajes.

2. Warsawer Zeitung (Periódico de Varsovia), (25 de octubre) miércoles 6 de noviembre de 1861, pg.3, Sección de misceláneos.

3. Botanische Zeitschrift - Gemeinnütziges Organ (Revista Botánica - Órgano sin fines de lucro) de Viena, N¹2, diciembre de 1861, pg.409. Sección de avisos personales.

4. Gartenflora Algemeine Monatschrift (Revista mensual general), dedicada a estudios florales y de jardinería en Alemania, Rusia y Suiza, y órgano de la Asociación Hortícola Imperial Rusa en San Petersburgo, 1862, pg. 167. Sección de notas personales y novedades.

\footnotetext{
${ }^{1}$ Hilje Quirós, Luko. "Juan Rafael Mora y las ciencias naturales en Costa Rica" Editorial Universidad Técnica Nacional, 2015. San José: Costa Rica. ISBN 9968629189, 9789968629188. $110 \mathrm{p}$.
} 
En Deutches Magazine für Garten und Blumenfunde (Revista Alemana de Jardinería y Hallazgos Florales), edición 1962, pg. 162, se publicó una nota destacando el trabajo que Eduard Sell llevaba a cabo en zonas tropicales, a riesgo de su propia salud, en nombre y por cuenta del Sr. Consejero Comercial Robert Schöller en Dürren (Prusia del Rin). Sell se había desempeñado anteriormente como Jefe del Jardín Blaß’chen en Elberfeld, y posteriormente en el Real Jardín Zoológico de Bruselas, cuyo director fue Jean Linden, en los que adquirió un conocimiento sólido de las plantas.

Esta última publicación destaca también el espíritu altruista del Sr. Schöller en pro de la investigación, y da una lista de plantas que estarán disponibles tan pronto como crecieran y fuera posible determinar cuántos especímenes serían prescindibles, y cuáles se podrían preparar y de cuántos emitir listas con precios, que se publicarían por ese mismo medio.

Es de hacer notar un detalle que ha sido destacado por muchos autores, acerca del extraordinario cuidado que estas revistas y otras ponían en la calidad artística de dibujos de especímenes, la mayoría de origen tropical, y que constituyen una suerte de ciencia y arte al mismo tiempo.

En 1864 Eduard Sell decide romper con Robert Schöller, y escribe a su padre una emotiva carta en la que comunica su decisión definitiva de establecerse en Costa Rica, al tiempo que le pide el envío de una lista de semillas.

En Gartenflora Algemeine Monatschrift (Revista Mensual General), para Alemania, Rusia y Suiza. 1873, pg. 320, aparece posible un intento por forzar el regreso de Eduard Sell a Alemania. Se menciona que años atrás viajó con la intención de importar plantas de Centroamérica, y que durante mucho tiempo desapareció sin dejar rastro, a pesar de muchos esfuerzos para descubrir dónde estaba, y que en fecha reciente había dado una señal de vida de San Ramón, en América Central, según la cual se encontraba bien y tenía la intención de volver a Europa pronto con un transporte de plantas raras. La nota la firma EM, posiblemente E. Mayer, Inspector del Jardín en Carlsruhe, cuyo nombre aparece en la portada de la revista. 
Para entonces, Eduard Sell había entablado una relación afectiva con la prusiana Wilhelmine Steffen en San Ramón de Alajuela, y procreado a Arturo Eduardo Sell Steffen, bisabuelo del autor de este artículo y nacido el 15 de setiembre de 1868 . El 16 de enero de 1874 nacerá Ernesto Eduardo Sell Steffen, fallecido 3 años después, cuyos padrinos de bautismo fueron Agustín Endrés y Agustina Piepper.

Augustus R. Endrés (1838-1874), originario de Alsacia y naturalizado norteamericano, se convirtió en una figura legendaria en la exploración botánica de Costa Rica e indudablemente en el más importante colector de orquídeas que alguna vez visitó los países centroamericanos ${ }^{2}$.

Una de las orquídeas miniaturas de Endrés será conocida como Lepanthes Selliana Endrés ex Luer, llamada así muy posiblemente en honor, según Luer, a un amigo o recolector colaborador de Endrés, apellidado Sell.

\section{El artículo}

\section{Semanario}

de la Asociación para la Promoción de la Horticultura en los Estados Reales de Prusia para la Jardinería y Botánica

Editado por el secretario general de la asociación, Profesor Dr. Karl Koch

№32, Berlín, 9 de agosto de 1862

La Extracción de caucho en Costa Rica

Por Eduard Sell, ahora en Costa Rica

Los artículos de exportación que posee la República de Costa Rica hasta el día de hoy, además del café, son muy pocos; desde 1860, sin embargo, se ha agregado el caucho, pero

\footnotetext{
${ }^{2}$ Ossembach, Carlos et al. Orchid Itineraries of Augustus R. Endrés in Central America: a Biographic and Geographic Sketch. Lankesteriana 10(1), p.19. 2010
} 
lamentablemente las exportaciones se han debilitado nuevamente debido a la repentina caída de los precios. Ya en 1850 se intentó extraer caucho de la savia de ciertos árboles. Para ello algunos empresarios se trasladaron a las riberas de los ríos Sarapiquí y San Carlos, donde estos árboles crecen en gran número. Desafortunadamente, carecían de conocimientos precisos sobre cómo preparar caucho. Así que uno no debería sorprenderse si las cosas fallaron. En lugar de caucho, todo lo que se obtuvo fue una masa espesa y grasosa de aspecto alquitranado y mal olor, que por lo tanto era completamente sin valor e inútil.

Cuando en 1860 los precios del caucho en los Estados Unidos de Norteamérica alcanzaron tal nivel que su extracción prometía ser un negocio muy rentable, en Costa Rica comenzó nuevamente a prestarse la atención a esta industria. Se comenzó el asunto con más conocimiento y de hecho se obtuvo, a un costo comparativamente bajo, un producto bastante bueno con el que ciertamente se podía contar para vender.

En todas las partes bajas de la república donde había árboles de caucho, los experimentos pronto asumieron una mayor extensión. En todas partes donde la preparación se abordó con experiencia, se tuvo éxito. Generalmente se cree que los árboles de la costa atlántica, así como los de las orillas de los ríos San Juan y Sarapiquí, contienen mucho más caucho que los de las orillas del San Carlos y de la provincia de Guanacaste ${ }^{3}$. Pronto se descubrió que tipos muy diferentes de árboles contienen caucho.

En Sudamérica se sabe que se obtiene principalmente de Hevea guianensis (Jatropha elastica) [hoy Hevea brasiliensis], mientras que en Costa Rica Castilloa elastica es el árbol que se suele utilizar para este fin. Mientras que el primero es una Euphorbiacee, el segundo, según la excelente investigación de Trecul ${ }^{4}$, pertenece a la familia Artocarpeae

\footnotetext{
${ }^{3}$ NT. Guanacarte, en el original.

${ }^{4}$ NT. Muy posiblemente Auguste Adolphe Lucien Trécul (o Auguste Trécul) (1818-1896). Botánico francés. Investigador en el Museo Nacional de Historia Natural de Francia y en el Ministerio de Agricultura de Francia. Se especializó en anatomía, fisiología, y organogenia vegetal.
} 
[hoy Moraceae]. Por tanto, ambos árboles son suficientemente diferentes. Castilloa elastica también ya está en el mercado y fue presentada por Linden en Bruselas. Allí también está disponible continuamente.

Los costarricenses Ilaman a este último Hule (pronunciado Uhle), mientras que los indios lo llaman Tassadusa (de Tassa, savia de caucho, y Dusa, árbol). Crece en grupos en suelos muy ricos en humus y negros, en zonas muy húmedas, preferiblemente cerca de ríos. La época de floración comienza en abril y dura hasta julio, pero la producción de caucho es independiente de ésta, así como de la temporada; por otro lado, se dice que la posición de la luna tiene una gran influencia, en la medida en que con lunas crecientes debe haber más caucho, con lunas decrecientes; por otro lado, se supone que hay más extracto acuoso presente en la savia lechosa. Cuanto más viejo es el árbol, más látex ${ }^{5}$ contiene y, por tanto, también caucho. En un árbol de tamaño mediano, de unos cuatro pies de diámetro, como se encuentra más comúnmente, se calculan entre doce y quince galones de látex (dos galones hacen un Maas alemán ${ }^{6}$ ); los árboles más gruesos a veces producen hasta 20 galones. Es importante que el mismo árbol puede ser explotado nuevamente después de 6 meses, pero en este caso la savia contiene menos caucho. Los árboles de menos de dos pies de diámetro se desangran y mueren. Por lo tanto, el gobierno tiene estrictamente prohibido utilizar árboles de menos de $2 \frac{1}{2}$ pies de diámetro para fabricar caucho ${ }^{7}$.

Mucho antes de que se abordara la producción de caucho en Costa Rica, los nativos lo hacían para sus propios fines. Utilizaron un método peculiar que se había mantenido en secreto durante mucho tiempo. Lo principal en la preparación es hacer que el látex cuaje rápidamente de tal manera que la sustancia de caucho se separe fácilmente de los otros componentes, especialmente de las sustancias extractivas. Para ello, los isleños de La

\footnotetext{
${ }^{5}$ NT. En alemán se utililza la palabra Milchsafte = jugos lechosos, que se traduce por savia lechosa. No obstante, el vocablo más apropiado parece látex.

${ }^{6}$ NT. Medida alemana no determinada.

${ }^{7}$ NT. Op. cit. Hilje Quirós, Luko.
} 
Mosquitia añaden la savia de una enredadera que ha sido diluida con agua, que probablemente pertenece al género Convolvulus.

Según el método habitual, se cubren piedras o tablas con finas capas del látex y esta operación se repite varias veces. Cuando la última capa se ha secado, el tablero o piedra se sumerge en la savia obtenida y la coagulación se produce inmediatamente. Probablemente se agregue agua fresca y clara al látex hasta que todo el extracto soluble se haya combinado con él y pueda eliminarse fácilmente. Para favorecer la disolución, en Nueva Granada se agrega un poco de brandy al agua. La coagulación ocurre aún más rápido si se agrega alumbre, es decir, $1 / 2$ libra por centímetro de agua.

Tan pronto como se encuentra en la selva virgen un lugar adecuado con un número suficiente de árboles y el agua necesaria, sobre todo los trabajadores, que se han provisto con suficiente comida y los utensilios necesarios para una estadía más larga, construyen una cabaña para su departamento, y un edificio abierto, pero con buen techado, para la preparación de caucho. Se utilizan mucho las hojas de musas y heliconias. Uno de los trabajadores, el Bejuquero ${ }^{8}$ (enredadera) sale a buscar la enredadera mencionada anteriormente. Los zarcillos más fuertes se cortan y golpean con un martillo de madera, antes de echarlos en agua pura y macerarlos aquí hasta que la savia esté completamente extraída. Ahora todo el líquido se filtra a través de un material de algodón y, por lo tanto, se limpia de sustancias extrañas y extractoras.

El látex se extrae mejor desde donde comienzan las ramas, que suele ser el caso a una altura de 20 a 30 pies. Por otro lado, algunos prefieren hacer las incisiones a una altura de 12 pies, creyendo que la savia es más gruesa en el fondo y por lo tanto contiene más goma. Las incisiones se efectúan a través de la corteza, de apenas media pulgada de espesor hasta la madera, están espaciadas uniformemente entre sí en la parte superior, pero convergen en dos en el extremo inferior, y suelen tener de 1 a 11\%2 pies de largo. El látex que fluye corre sobre la hoja de un cuchillo o cualquier superficie de hierro algo cóncava

${ }^{8}$ NT. Lit. 
hasta un recipiente debajo. Se obtiene un promedio de 2 galones en 10 minutos. El líquido obtenido de esta forma se limpia mediante un tamiz fino, que se debe agitar vigorosamente a mano. Para no tener pérdidas en el residuo, a menudo se agrega agua dulce. Lo principal es que todo esto ocurra lo más rápido posible, porque la fermentación comienza a los pocos días y el látex se echa a perder.

El látex purificado se vierte en recipientes poco profundos y se le proporciona inmediatamente la adición necesaria de la savia rastrera. Aquí, sobre todo, se debe observar la medida correcta, lo que requiere no poca práctica. Dependiendo de la concentración del látex, a veces hay más, o a veces menos para agregar. Demasiada agua provoca demasiada coagulación, por lo que la goma pierde su elasticidad, mientras que, al revés, hace que el látex cuaje lentamente o no cuaje. También se debe tener mucho cuidado para asegurarse de que no caigan gotas de lluvia a través del haz de la hoja hacia el látex, que ya se ha mezclado con la savia rastrera, y que esto evita la coagulación. Después de unos 15 minutos se remueve bien con un palo pequeño, luego se golpea el recipiente con la mano y se le mantiene girándolo. Si en el líquido marrón se empieza a formar una especie de torta, se debe poner la mano encima y presionarla un poco más fuerte, para que esta última se vuelva más consistente. Cuando la torta ha adquirido una forma bastante plana y, por tanto, ha alcanzado la firmeza requerida, se coloca sobre una tabla plana y se prensa aún más mediante un rodillo de 100-150 libras, para separar los extractos que todavía están en el interior y se expulsen por completo.

Esto finaliza el procedimiento. Las tortas se sellan por el fabricante y se colocan una encima de la otra. Hacia la tarde se ponen en cestas que se cubren con juncos y luego se cuelgan en agua corriente. Aquí las tortas se bañan constantemente en agua. Esto es necesario para que durante la contracción del caucho la sustancia extractiva, que todavía no está completamente separada, se lave rápidamente. A los dos días, se sacan las cestas del río y se extienden las tortas en unas ligeras rejillas fijadas bajo el techo, adquiriendo paulatinamente la firmeza necesaria. Demasiado calor es muy perjudicial y detiene el 
secado. Incluso a una temperatura superior a los $18^{\circ} \mathrm{R}^{9}$, el caucho se vuelve grasoso y alquitranado.

Las láminas de caucho así preparadas a partir del látex de Castilloa elastica se ven completamente blancas al principio; sólo después de un largo período de almacenamiento adquieren un color marrón rojizo. Se debe tener cuidado de que las láminas individuales no se coloquen una encima de la otra demasiado pronto, porque la exudación de la sustancia extractiva continuará durante mucho tiempo, aunque en menor medida. Si esto no sucede, la goma se pudre fácilmente. La sustancia extractiva de color marrón oscuro que permanece en el líquido al principio es un tinte, por lo que las manos de los trabajadores se ven marrones durante mucho tiempo.

De acuerdo con el método anterior, donde no se usó el agua de la enredadera, se usan recipientes cilíndricos de 4 a 6 pies de alto, con una espita en la parte inferior, y que pueden contener de 30 a 50 medidas de líquido. Después de purificar la savia, se le agregan cuatro partes de agua pura, después de lo cual se agita vigorosamente. Después de permanecer quieta durante 24 horas, la goma se deposita en la parte superior y la sustancia extractiva se desprende hacia abajo, después de lo cual se escurre mediante el pasador situado debajo. Nuevamente, se vierte agua fresca sobre el látex que ya se ha cuajado y esto se repite hasta que la torta tenga un color café. La masa se coloca ahora sobre un paño extendido sobre arena, se deja reposar 10 a 15 días, tiempo durante el cual la sustancia extractiva se asienta y es absorbida por la arena. Finalmente, como en el método anterior, se lleva las láminas redondas al agua corriente durante 3 días y finalmente se dejan secar a la sombra. Las tortas probablemente también se colocan debajo de un rodillo (prensa) para exponerlos a una presión uniforme por un periodo de 24 horas.

\footnotetext{
${ }^{9} \mathrm{NT} .18$ grados Reaumur $=22.5$ grados Celsius. Para convertir los grados Réaumur $\left({ }^{\circ} \mathrm{R}\right)$ en grados Celsius $\left({ }^{\circ} \mathrm{C}\right.$ ), el respectivo valor debe multiplicarse por 1,25 (es decir, $C o=R \circ$ 5/4).
} 
El rendimiento de caucho puro fluctúa, como se indicó anteriormente, según la edad de los árboles, el punto de corte y el método de preparación. Sin embargo, en promedio se obtiene la cuarta parte del peso, y rara vez la tercera. El caucho de Costa Rica, elaborado con el método húmedo mencionado anteriormente, es una de las mejores variedades en los mercados de Londres y Nueva York.

Dado que los precios cayeron repentinamente a fines del año pasado, lamentablemente varios empresarios han abandonado el negocio nuevamente. El año pasado se exportaron alrededor de 1.500 Centner $^{10}$ de caucho de Greytown y Matina y 300 Centner de Puntarenas $^{11}$. Dado que el número de árboles en Costa Rica es muy significativo, el pequeño país puede en el futuro proporcionar cantidades aún mayores de este útil producto. El único inconveniente es que el trabajo solo se puede realizar en las zonas más insalubres del país, donde incluso los nativos que viven en los valles altos no pueden quedarse mucho tiempo. Un europeo inmigrante será aún menos capaz de tolerar este clima.

Los editores ${ }^{12}$ se permiten luego comentar sobre la planta madre del caucho centroamericano, sobre todo porque tiene una copia a su disposición, que el jardín botánico de Berlín debe a la amabilidad del director Linden en Bruselas. Castilloa elastica Cerv. crece no solo en México y en varias islas de las Indias Occidentales, sino que parece extenderse por toda América Central. Allí le encantan las zonas más bajas con un clima cálido y seco. Esta circunstancia puede dificultar especialmente que los europeos presten la atención necesaria a la preparación del caucho, pues mientras difícilmente los indios, que están más acostumbrados al clima local, pueden soportar largos períodos en las malsanas tierras bajas, los europeos suelen morir rápidamente de fiebre amarilla. Por esta

\footnotetext{
${ }^{10}$ NT. Centner = Unidad de peso que se utilizaba en especial en Europa y normalmente equivale a unas 110 libras (unos 50 kilogramos).

${ }^{11}$ NT. Punta Arenas, en el original.

12 NT. A partir de aquí empieza una nota de los editores.
} 
razón, la producción del caucho sigue siendo demasiado costosa y solo parece valer la pena si el precio del producto no es tan bajo.

A diferencia de otras plantas en climas cálidos, Castilloa elastica crece gregariamente y ocupa particularmente las orillas planas de los ríos desde la desembocadura hasta donde emergen de las montañas. Ya fue descrito e ilustrado a fines del siglo anterior por el entonces profesor de botánica en México, Cervantes ${ }^{13}$, en un tratado especial (De Resina Elastica et de Arbore Novae Hispaniae Resinam Hujusmodi Producente) como suplemento del periódico literario del 2 de julio de 1794. No sabemos quién fue Castillejo, de quien toma prestado el nombre.

Hasta hace poco, la posición de la planta era algo dudosa, hasta que finalmente Trécul la describió de nuevo en su excelente monografía sobre Artocarpaeae (Ann. D. Sc. Natur. 3. sér. T. VIII, p. 136). Según esto, pertenece a la familia que acabamos de mencionar, es decir, a la división de Olmedieen. Parece estar adquiriendo un gran tamaño en la patria, porque los árboles con un diámetro de 9 pies no son infrecuentes. La corteza es lisa, mientras que las ramitas y las ramas más jóvenes están cubiertas de pelo amarillo pálido, al igual que la superficie inferior de las hojas, que suelen medir más de 1 pie de largo, pero solo la mitad de ancho y en forma de corazón. Las flores separadas se encuentran en la misma planta en diferentes campos de flores (receptáculo), que están rodeados por una cubierta en forma de copa.

La otra planta madre del caucho americano mencionada en el artículo, Hevea guianensis Aubl., bajo el nombre Siphonia elastica Pers. más conocido, crece con mucha frecuencia en la Guayana y suele proporcionar el producto mencionado anteriormente. La planta no crece tan grande y en promedio alcanza una altura de 50 a 60 pies con un diámetro de

\footnotetext{
${ }^{13}$ NT. Muy posiblemente se refieran a Vicente de Cervantes, "De resina elastica generatim, et de arbore Novae Hispaniae resinam hujusmodi producente, speciatim". Suplemento a la Gazeta de literatura, México 2 de julio 1794.
} 
tronco de $2 \frac{1}{2}$ pies. En la punta de las ramas se encuentran los folíolos elípticos, de color blanco grisáceo y sin pelo en los tallos expandidos en la base.

Figura 1. Encabezado de la publicación que contiene el artículo traducido.

\section{Wochenschrifit}

des Vereines zur Beförderung des Gartenbaues in den Königlich Preussischen Staaten

\section{Gärtnerei und Pflanzenkunde. \\ für}

Redigire

von dem General-Sekretair des Vereines.

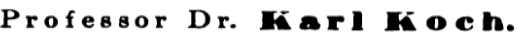

No. 32.

Berlin, den 9. August

1862.

Preis des Jahrganges $5 \frac{1}{\mathrm{j}}$ Thlr.. sowohl bei Bezug durch den Buchbandel. als auch franco durch alle Post-Anstaiten aes deutsch-бsterreichischen Post-Vereinb.

Inhalt: Die Kautschuck-Gewinnung in Costa-Rica. - Ein Ausflug nach Belgien und nach den Niederlanden im ersten Frahjahre 1862 (Fortsetzung). - Beilage.

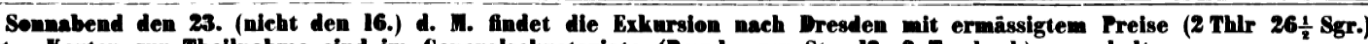
statt. Karten zur Theilnahme sind in Ceneralsekretariate (Bernburger Str. 13. 2 Tr. hoch) su erhalten.

Fuente: Bayer Staatsbibliothek. Disponible en Google ${ }^{\text {TM }}$ books.

Figura 2. Castilloa Elastica. Rama con fruto.

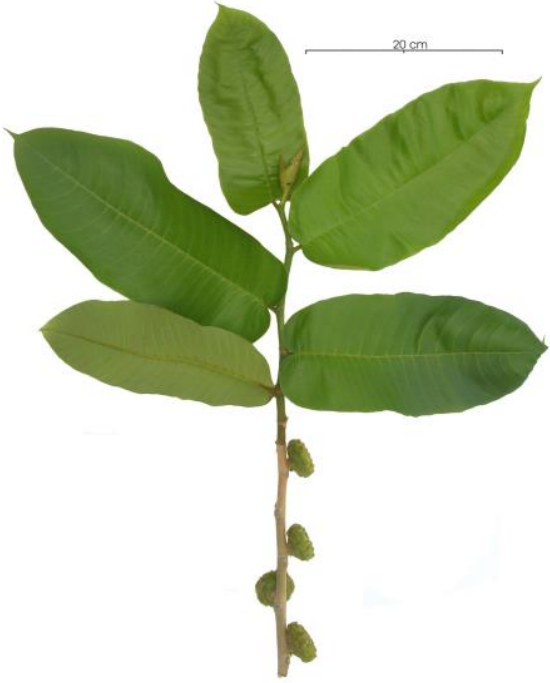

Fuente: Environmental Sciences Program, Smithsonian (C) Tropical Reserarch Institute.

Figura 3. Árboles de Castilloa Elastica creciendo en un hábitat nativo. Los monos aulladores apenas pueden ser vistos en el árbol, comiendo los frutos. 


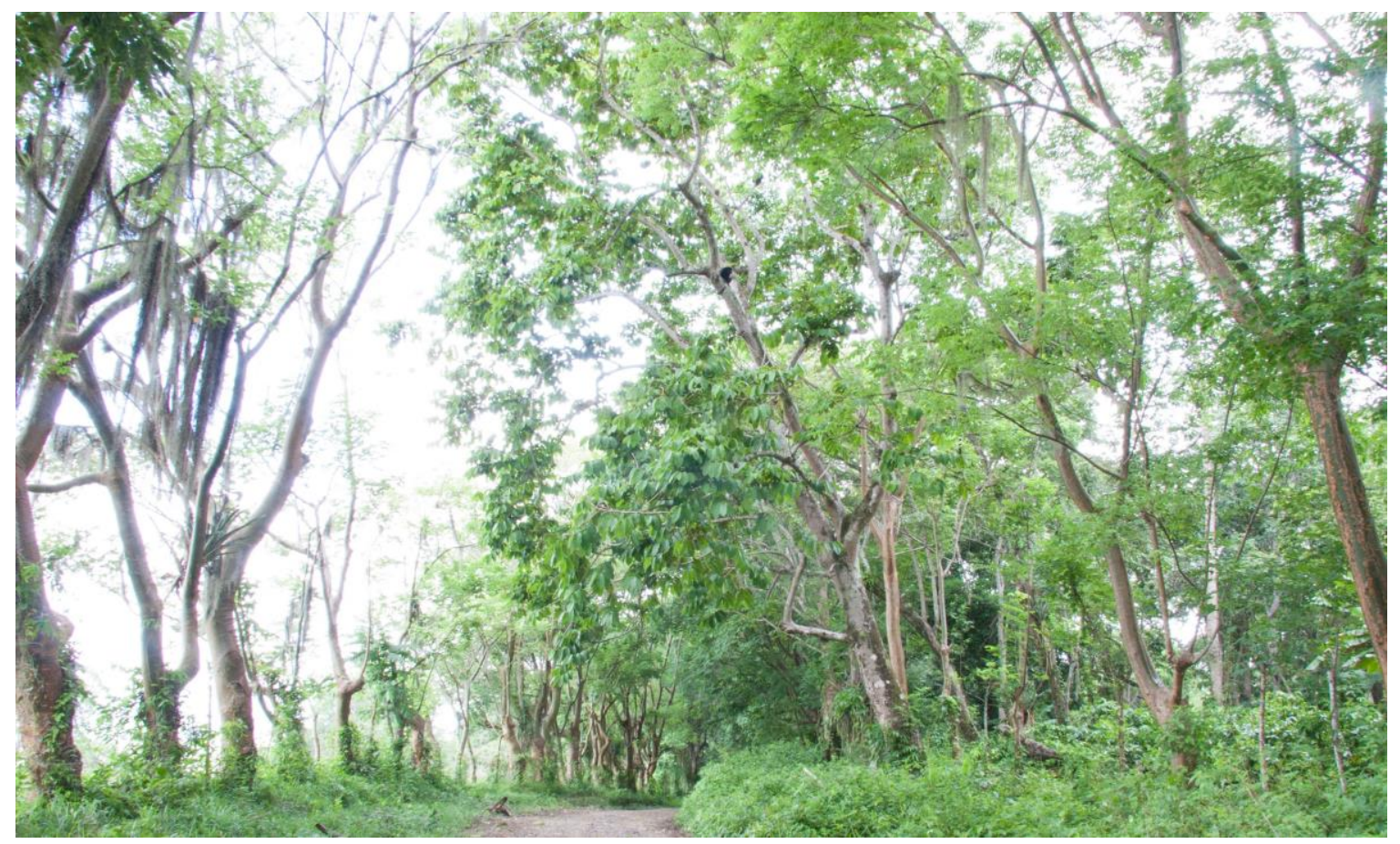

Fuente: O. M. Montie. (C) Creative Commons.

\section{Conclusiones del traductor}

El artículo de Eduard Sell sobre la extracción de caucho en Costa Rica, publicado en Berlín en 1862 , parece estar dirigido a buscar personas interesadas en el viejo continente, en una actividad que, si bien enfrentaba altibajos y escasa oportunidad en ese momento preciso, recobraría el vigor de un buen negocio si el precio internacional de esa materia prima aumentaba en los mercados de Londres y Nueva York.

No es extraño encontrar fuera de las fronteras de Costa Rica trabajos llevados a cabo por viajeros y visitantes extranjeros, quienes llegaron con muy diversas motivaciones a explorar y estudiar los especímenes de flora y fauna del país. Hubo quienes no llegaron a publicar tratados completos sobre temas determinados, dado que su participación tuvo como fin sentar las bases de negocios comerciales entre las regiones tropicales y sus países de origen, y que una vez establecidos volvieron a su tierra natal. Otros establecieron vínculos afectivos fuertes, que los llevó a modificar su objetivo original y a establecerse en el país formando familias y nuevos círculos de amistad e intereses Tal es el caso de mi tatarabuelo Eduardo Sell Modes. 


\section{Agradecimientos}

Agradezco la invaluable ayuda del Dr. Luko Hilje Quirós, cuyo criterio científico permitió depurar la nomenclatura de especies en su evolución diacrónica, y otros aspectos técnicos. Además, porque al compartírselo, me motivó para que lo publicara. También al Dr. Óscar Alvarado Vega por sus observaciones de composición.

\section{Bibliografía}

Hilje L. 2015. Juan Rafael Mora y las ciencias naturales en Costa Rica. San José, Costa Rica. San José: Costa Rica. EUTN.

Ossembach C. et al. 2010. Lankesteriana - International Journal of Orchidology: Orchid Itineraries of Augustus R. Endrés in Central America: a biographic and Geographic Sketch. San José: COSTA RICA. UCR. 